Report

\title{
p53 protein accumulation in non-invasive lesions surrounding $p 53$ mutation positive invasive breast cancers
}

\author{
Susan J. Done ${ }^{1,3}$, Nona C.R. Arneson ${ }^{3}$, Hilmi Özçelik ${ }^{1,3}$, Mark Redston ${ }^{1,3}$, and Irene L. \\ Andrulis $1,2,3$ \\ ${ }^{1}$ Departments of Laboratory Medicine and Pathobiology, ${ }^{2}$ Department of Molecular and Medical Genetics, \\ ${ }^{3}$ Samuel Lunenfeld Research Institute, Mount Sinai Hospital, The University of Toronto, Toronto, Ontario, Canada
}

Key words: breast cancer, DCIS, hyperplasia, mutation positive cases, p53 protein

\begin{abstract}
Summary
p53 mutation is a common event in sporadic breast cancer being found in $15-50 \%$ of invasive carcinomas. The purpose of this study was to determine the earliest histologic stage at which $p 53$ mutation could be detected with a widely used anti-p53 antibody (DO7, Novocastra) which recognizes both wild type and mutant forms. p53 expression was assessed immunohistochemically in 12 primary breast carcinomas with known $p 53$ mutations and in all pre-malignant epithelial lesions surrounding these invasive cancers. Strong p53 nuclear staining was found in all of the tumors known to have missense mutations and none of the tumors with truncation mutations. In cases with intense staining in the invasive carcinoma, a similar quality of staining was also seen in all areas of DCIS (ductal carcinoma in situ) and was representative of missense p53 mutations. Lighter nuclear staining intensity was observed in up to $40 \%$ of cells in areas of hyperplasia and in up to $30 \%$ of normal breast lobules irrespective of the type of mutation found in the invasive carcinoma. This weak staining was not specific to mutated p53 and may indicate increased amounts of normal p53 protein.

We conclude that p53 inactivation occurs prior to invasion in breast carcinogenesis, with mutations being uniformly identified in DCIS associated with p53-mutated invasive carcinomas. In contrast, there is no evidence that epithelial hyperplasia or epithelial cells of the terminal duct lobular unit harbor the same mutations as their associated invasive carcinoma.
\end{abstract}

\section{Background}

Mutation in the $p 53$ gene is one of the most frequent somatic genetic events in breast cancer [1], as it is in many other human malignancies. The normal cellular functions of p53 include cell cycle regulation and control of entry into apoptosis. Mutation of p53 has been proposed as a critical event in the development of the genomic instability necessary for the accumulation of mutations in other genes during neoplastic progression. p53 protein has a very short half-life and does not normally accumulate in cells. However, mutations in the gene can result in a conformationally altered nuclear protein, increasing stability and resulting in positive immunohistochemical staining. Immunohistochemistry is a useful tool to examine p53 status because many different types of epithelial lesions can be evaluated simultaneously. Although immunohistochemical accumulation of p53 protein has often been used as an indicator for the presence of a p53 mutation, this approach may lead to a significant number of false-positives and false-negatives. Studies of immunohistochemical staining supported with analysis of p53 DNA mutational status are necessary to evaluate the role of $\mathrm{p} 53$ alterations in breast cancer progression.

The purpose of this study was to identify the earliest histologic stage of breast carcinogenesis at which $p 53$ mutations could be detected by immunohistochemistry in a group of cases known to harbor sporadic p53 mutations in their invasive components from previous mutational analysis. The approach taken is similar to that used in previous studies; 
finding invasive cancers known to harbour genetic alterations and identifying which surrounding 'early' lesions are linked by the same genetic alteration. From a group of 146 node negative breast cancers we identified 12 that contained mutations, as detected by SSCP and sequence analysis, and had blocks available for immunohistochemistry. We have previously characterized the p53 mutational status of 68 epithelial proliferations and normal lobules surrounding seven of these invasive carcinomas. To understand the observed staining patterns we determined the spectrum of $p 53$ mutations that can be recognized by a widely used monoclonal anti-p53 antibody (DO7, Novocastra) that recognizes an epitope at amino acids $21-25$ of p53 [3]. In this report we describe the p53 immunohistochemical staining patterns seen in normal mammary epithelium, epithelial hyperplasias and areas of ductal carcinoma in situ surrounding invasive carcinomas known to harbor $p 53$ mutations.

\section{Materials and methods}

\section{Selection of cases}

Tissue samples were obtained from 146 primary breast carcinomas resected at Mount Sinai Hospital, Toronto, Canada between 1987 and 1992. As part of an ongoing prognostic study, p53 mutational status was determined in snap frozen tumor samples from women with no evidence of metastatic disease as determined by axillary lymph node status and clinical staging at the time of surgery. A group of 12 cases known to contain sporadic $p 53$ mutations (six missense, six nonsense) that also had tissue blocks available for immunohistochemical study were selected. Eleven of the 12 are from a group of 17 cases subjected to SSCP and sequence analysis of the $p 53$ gene in a previous study. The patients were between 36 and 69 years of age at the time of surgery with tumors $0.1-2.5 \mathrm{~cm}$ in diameter. The tumors were all of ductal histological type and modified Bloom - Richardson grade II or III.

A total of 47 blocks were selected for immunohistochemical analysis, ranging from one to nine per case. Sections were selected based on the presence of areas of hyperplasia or DCIS. A total of 166 areas of DCIS (range 0-72 per case), 3 areas of florid hyperplasia (range $0-3$ per case), 33 areas of moderate hyperplasia (range 0-12 per case), 39 areas of mild hyperplasia (range $0-16$ per case) and 481 normal ducts or lobules (range 0-147 per case) were studied.

\section{Immunohistochemical staining}

Immunohistochemistry was performed in the Department of Pathology and Laboratory Medicine at Mount Sinai Hospital. Formalin-fixed paraffin-embedded tissue sections $(5 \mu \mathrm{m})$ were placed on aminopropyltriethoxysilane (Sigma Chemical Co., St. Louis, MO) coated slides. Sections were immersed in a citrate buffer (10 mM, pH 6.0) and heated to boiling in a microwavable pressure cooker. Endogenous peroxidase activity was inactivated with hydrogen peroxide, and the sections were blocked with a protein blocker (Signet Laboratories Inc., Dedham MA). The selected sections, together with appropriate controls, were incubated for $1 \mathrm{~h}$ with a mouse monoclonal antibody to p53 (DO7 clone; Novocastra Laboratories Ltd., Newcastle, England). After washing, the sections were incubated with biotinylated-horse anti-mouse IgG, followed by avidin-biotin peroxidase complex (Elite kit; Vector Laboratories, Burlingame, CA), and developed with diaminobenzidine (DAB; Sigma Chemicals, St. Louis, MO). Sections were counterstained with hematoxylin.

\section{Scoring system}

One observer scored all slides. Nuclear staining of epithelial cells was considered as positive. The percentage of positive cells in each type of lesion was estimated from $0 \%$ to $100 \%$. For each positively staining area, the intensity of nuclear staining was evaluated semi-quantitatively on a four point scale as follows: $0=$ no staining; $1=$ light staining; $2=$ staining of moderate intensity; $3=$ intense staining.

\section{Results}

Relationship between immunohistochemistry and SSCP/sequence analysis in invasive carcinoma

Six of the twelve invasive carcinomas harbored truncation mutations in the $p 53$ gene and six tumors had missense mutations as shown in Table 1. The carcinomas containing missense mutations showed intense staining in the nuclei of invasive carcinoma cells (Figure 1A). In cases with strong staining in the invasive components, the staining was diffuse and uniform in intensity throughout the tumor. Intense staining was only observed in nuclei. The six carcinomas with truncation mutations did not show any areas of intense staining. 
Table 1. p53 mutations and immunohistochemical staining in invasive carcinoma

\begin{tabular}{rllll}
\hline $\begin{array}{l}\text { Case } \\
\text { no }\end{array}$ & $\begin{array}{l}\text { Exon } \\
\text { containing } \\
\text { mutation }\end{array}$ & Mutation & $\begin{array}{l}\text { Effect on } \\
\text { protein }\end{array}$ & $\begin{array}{l}\text { p53 } \\
\text { staining } \\
\text { intensity }\end{array}$ \\
\hline 490 & 5 & TGC $>$ TAC codon 176 & Cys $>$ Tyr & 3 \\
814 & 5 & CGC $>$ GGC codon 175 & Arg $>$ Gly & 3 \\
1857 & 5 & TTT $>$ TTG codon 134 & Phe $>$ Leu & 3 \\
2005 & 5 & CGC $>$ CAC codon 156 & Arg $>$ His & $3^{*}$ \\
387 & 7 & GAA $>$ AAA codon 258 & Glu $>$ Lys & 3 \\
992 & 7 & ins CCA codon 251 & In frame & 3 \\
775 & 4 & del A codon 94 & Truncated & 0 \\
1085 & 4 & TAC $>$ TAA codon 107 & Truncated & 0 \\
2081 & 4 & Del G codon 117 & Truncated & 0 \\
2649 & 5 & Del A codon 139 & Truncated & 0 \\
1765 & 6 & CGA $>$ TGA codon 213 & Truncated & 0 \\
2262 & 7 & ins GTTA codon 236 & Truncated & 0 \\
\hline
\end{tabular}

* The original area of invasive carcinoma that was analyzed had been cut through. The same mutation could be detected in microdissected areas of DCIS.

\section{Results of immunhistochemical staining in} pre-invasive, pre-neoplastic and benign epithelium

Pre-invasive lesions were observed in all six of the cases with missense mutations as described in Table 2. In five of these cases, intense staining (score 3 out of 3) was found in 29/29 separate areas of carcinoma in situ but was not observed in 40/40 separate areas of hyperplasia (Table 2 and Figure 1B).

In one of our cases, (case 2005), invasive cancer was not available for study, as the invasive cancer was small and had been cut through at the time of initial diagnosis. A missense mutation was found in multiple microdissected areas of micropapillary DCIS even though only focal intense staining involving a portion of the cells lining a dilated duct was seen (Figure 1C-E).

Intense staining was not detected in areas of hyperplasia, regardless of degree (mild, moderate or florid). In one case (case 814), an area of mild hyperplasia showed $10 \%$ positivity of moderate intensity (score 2 out of 3). Fifteen further areas of hyperplasia stained weakly positive (score 1 out of 3 ) with up to $80 \%$ of the cells stained. This type of weakly positive staining was seen in areas of hyperplasia from cases with either type of mutation (truncation or missense) in the invasive carcinomas (Tables 2 and 3). The degree (percentage of positive cells) or intensity of staining was not related to the distance of the lesion from the invasive cancer.
Four areas of adenosis were studied, and all showed staining of weak intensity (score 1 out of 3 ) in $10-60 \%$ of epithelial cells. Columnar metaplasia was found in one case (case 490) and showed weak intensity staining in up to $10 \%$ of epithelial cells. Cytoplasmic but not nuclear staining was seen in apocrine metaplasia.

Weak staining (score 1 out of 3 ) was detected in a minority of cells in normal lobules from cases with truncation mutations (Table 3 ), as well as from cases with missense mutations (Table 2). Again the degree or intensity of staining was not related to the distance from the invasive cancer. Two exceptions to the general staining pattern were found by immunohistochemical analysis (Table 2). In one case (case 1857, Table 2) strong staining (score 3 out of 3 ) was seen in a normal lobule immediately adjacent to an area of invasive carcinoma and in another case (case 490), focal staining of moderate intensity (score 2 out of 3 ) was found in the majority of cells within a segment of a normal lobule (Figure 1F). Four of the normal areas studied by immunohistochemistry had previously been analyzed by microdissection and SSCP and/or direct sequence analysis and only wild type $p 53$ had been detected (Table 4). One of these four normal areas was the lobule in case 490 showing moderate (score 2 out of 3 ) intensity staining in a portion of the terminal duct lobular unit. Microdissection of adjacent sections to the one used for immunohistochemistry was not possible in either case, as when we went back 

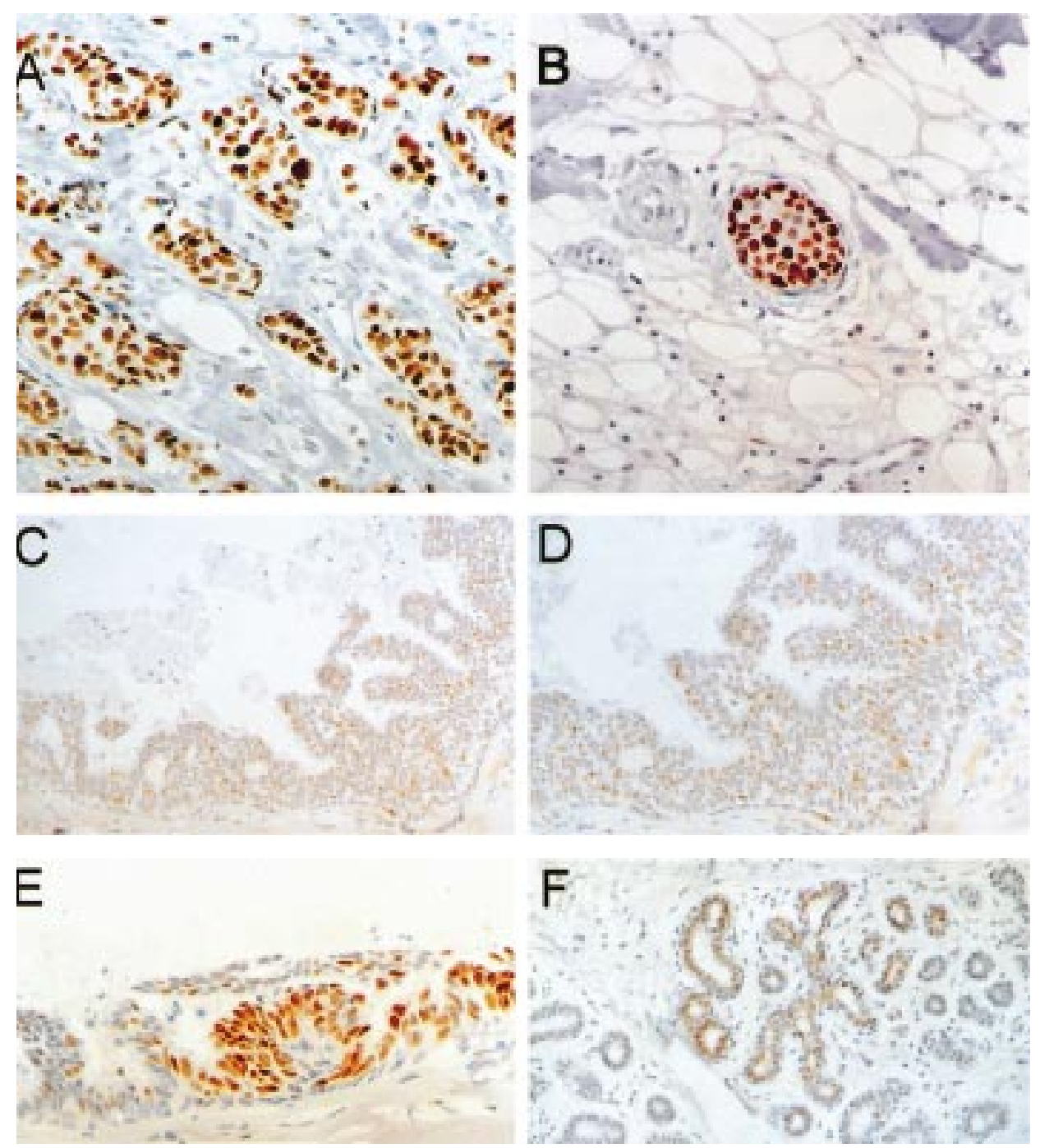

Figure 1. Immunohistochemical staining with an anti-p53 antibody (DO7 clone, Novocastra). (A) Area of invasive carcinoma from case 490 containing a missense mutation and showing strong intensity staining (score 3 out of 3); (B) Area of DCIS from case 1857 showing strong intensity (score 3 out 3) staining; (C) Micropapillary DCIS from case 2005 photographed at low power; (D) Twenty percent of cells stain with moderate intensity (score 2 out of 3 ) in micropapillary DCIS photographed at high power; (E) Area of focal strong staining (score 3 out of 3 ) in micropapillary DCIS; (F) The segment of the normal terminal duct lobular unit from case 490 showing moderately strong staining (score 2 out 3$)$.

to the blocks, sections immediately adjacent did not contain sufficient tissue for microdissection and PCR analysis.

\section{Discussion}

Evidence is accumulating that breast cancer, like colon cancer, develops via an accumulation of genetic events. Despite recent intense interest in the breast cancer genes $B R C A 1$ and $B R C A 2$, it is recognized that their role in breast cancer may be limited to familial breast cancer cases that comprise only about $5-10 \%$ of all breast cancer cases. Relatively few genes have been well characterized in sporadic breast cancer. One of the genes most frequently studied is the tumor suppressor gene $p 53$, which is mutated in 15$50 \%$ of sporadic breast cancers [1]. To investigate the timing of p53 mutation during neoplastic progression, we have studied a total of 722 benign, hyperplastic and pre-invasive neoplastic epithelial areas surrounding invasive breast cancers known to harbor $p 53$ mutations. 
Table 2. Immunohistochemical staining patterns observed in cases with $p 53$ missense mutations

\begin{tabular}{|c|c|c|c|}
\hline Case no & Lesion & $\begin{array}{l}\text { Percentage } \\
\text { positivity }\end{array}$ & $\begin{array}{l}\text { Intensity } \\
(1-3)\end{array}$ \\
\hline \multirow[t]{7}{*}{490} & $\mathrm{Inv} \mathrm{Ca}$ & 95 & 3 \\
\hline & DCIS & 95 & 3 \\
\hline & Mod HP & $30-40$ & 1 \\
\hline & Mild HP & $10-15$ & 1 \\
\hline & Adenotic nodule & $10-60$ & 1 \\
\hline & Columnar met & 10 & 1 \\
\hline & $\mathrm{N}$ & 10 & $1 *$ \\
\hline \multirow[t]{4}{*}{814} & $\mathrm{Inv} \mathrm{Ca}$ & 100 & 3 \\
\hline & DCIS solid & 100 & 3 \\
\hline & Mild hyperplasia & 10 & 2 \\
\hline & $\mathrm{N}$ & 30 & 1 \\
\hline \multirow[t]{3}{*}{1857} & $\mathrm{Inv} \mathrm{Ca}$ & 100 & 3 \\
\hline & DCIS solid & $10-80$ & 3 \\
\hline & $\mathrm{N}$ & 5 & $1 * *$ \\
\hline \multirow[t]{4}{*}{2005} & DCIS micropap & $10-20$ & $1-2^{* * *}$ \\
\hline & Pap lesion & $10-20$ & 1 \\
\hline & Adenosis & $10-20$ & 1 \\
\hline & $\mathrm{N}$ & $10-20$ & 1 \\
\hline \multirow[t]{4}{*}{387} & $\mathrm{Inv} \mathrm{Ca}$ & 90 & 3 \\
\hline & DCIS solid \& com & 80 & 3 \\
\hline & Mild HP & 80 & 1 \\
\hline & $\mathrm{N}$ & 10 & 1 \\
\hline \multirow[t]{4}{*}{992} & $\mathrm{Inv} \mathrm{Ca}$ & 60 & 3 \\
\hline & DCIS solid & 60 & 3 \\
\hline & $\mathrm{HP}$ & $0-10$ & 1 \\
\hline & $\mathrm{N}$ & 0 & \\
\hline
\end{tabular}

Inv Ca : invasive carcinoma; DCIS : ductal carcinoma in-situ; HP : hyperplasia; Mod : moderate; met : metaplasia; micopap : micropapillary; Pap : papillary; com : comedo; $\mathrm{N}$ : normal epithelium.

* Focal staining of moderate intensity $(2 / 3)$ is seen in a portion of a single normal lobule (Figure $1 \mathrm{~F}$ ), $80 \%$ of cells staining. ** $90 \%$ of cells stain with $3 / 3$ intensity in histologically normal lobules immediately adjacent to strongly staining invasive carcinoma.

*** A single area of micropapillary DCIS stains focally with $3 / 3$ intensity (Figure 1E).

Immunohistochemistry was used because of the ability to simultaneously study a large number of adjacent lesions from individual cases.

The relationship between p53 mutational status and immunohistochemical positivity has been examined previously, mainly in invasive cancers. While immunohistochemistry is seen as a useful screening tool by some $[4,5]$, it has been deemed unreliable by
Table 3. Immunohistochemical staining patterns in cases with $p 53$ truncation mutations

\begin{tabular}{|c|c|c|c|}
\hline Case no & Lesion & $\begin{array}{l}\text { Percentage } \\
\text { positivity }\end{array}$ & Intensity (1-3) \\
\hline \multirow[t]{5}{*}{775} & Inv $\mathrm{Ca}$ & 0 & \\
\hline & DCIS & $0-10$ & $1-2$ \\
\hline & Mod HP & 40 & 1 \\
\hline & Mild HP & 0 & \\
\hline & $\mathrm{N}$ & 10 & $1-2$ \\
\hline \multirow[t]{3}{*}{1085} & Inv Ca & 0 & \\
\hline & DCIS & 0 & \\
\hline & $\mathrm{N}$ & 0 & \\
\hline 2081 & $\mathrm{Inv} \mathrm{Ca}$ & 0 & \\
\hline \multirow[t]{3}{*}{2649} & $\mathrm{Inv} \mathrm{Ca}$ & 0 & \\
\hline & DCIS & 0 & \\
\hline & $\mathrm{N}$ & 10 & 1 \\
\hline \multirow[t]{3}{*}{1765} & Inv $\mathrm{Ca}$ & 10 & 1 \\
\hline & DCIS & 10 & 1 \\
\hline & $\mathrm{N}$ & 10 & 1 \\
\hline \multirow[t]{3}{*}{2262} & $\mathrm{Inv} \mathrm{Ca}$ & 0 & \\
\hline & DCIS & 0 & \\
\hline & $\mathrm{N}$ & $0-5$ & 1 \\
\hline
\end{tabular}

Inv $\mathrm{Ca}$ : invasive carcinoma; DCIS : ductal carcinoma in-situ; HP : hyperplasia; Mod : moderate; $\mathrm{N}$ : normal epithelium.

others [6]. Chakravarty et al., concluded that while there is a good concordance between immunohistochemistry and DNA analysis (PCR-SSCP and genomic sequencing), immunohistochemical detection of p53 was not an absolute indicator of mutation [5]. Despite the occurrence of false positives and false negatives, p53 immunohistochemical positivity with DO7 or monoclonal PAb1801 has been associated with an increased disease recurrence frequency in one study [7]. Furthermore, p53 immunohistochemical positivity was found to be an independent prognostic parameter in a large series of 942 breast cancer cases [8] and in a study of 256 cases with 6 years of followup [9]. This issue remains controversial, however, and other groups have been unable to demonstrate the prognostic utility of detection of p53 abnormality by immunohistochemistry [10]. Both Sjogren et al. [11] and Kovach et al. [12] found that they obtained more accurate prognostic information with cDNA sequencing than with immunohistochemistry using the monoclonal antibody PAb1801.

We found that the DO7 antibody was able to strongly stain tumor cells with p53 missense muta- 
Table 4. Immunohistochemical staining in microdissected lesions previously subjected to p53 mutational analysis (SSCP/direct sequencing)

\begin{tabular}{|c|c|c|c|c|}
\hline $\begin{array}{l}\text { Case } \\
\text { no }\end{array}$ & Histology & $\begin{array}{l}\text { Molecular } \\
\text { analysis }\end{array}$ & $\begin{array}{l}\text { Immuno- } \\
\text { positivity } \\
(\%)\end{array}$ & $\begin{array}{l}\text { Immuno- } \\
\text { intensity } \\
(1-3)\end{array}$ \\
\hline 490B & Mild HP & $\mathrm{W}$ & 15 & 1 \\
\hline G & Mod HP & $\mathrm{W}$ & 5 & 1 \\
\hline $\mathrm{H}$ & Mod HP & $\mathrm{W}$ & 40 & 1 \\
\hline I & Mod HP & W & 30 & 1 \\
\hline $\mathrm{J}$ & $\mathrm{N}$ lobule & $\mathrm{W}$ & 10 & $1^{*}$ \\
\hline $\mathrm{K}$ & Adenotic nodule & $\mathrm{W}$ & 10 & $1-2$ \\
\hline $\mathrm{N}$ & $\begin{array}{l}\text { Columnar met/ } \\
\text { adenosis }\end{array}$ & $\mathrm{W}$ & 10 & 1 \\
\hline $\mathrm{O}$ & $\mathrm{N}$ lobule & $\mathrm{W}$ & 0 & \\
\hline Q & Adenotic lobule & $\mathrm{W}$ & 60 & 1 \\
\hline $\mathrm{R}$ & Florid HP & $\mathrm{W}$ & 10 & 1 \\
\hline S & Mild HP & $\mathrm{W}$ & 10 & 1 \\
\hline $\mathrm{T}$ & Mod HP & W & 20 & 1 \\
\hline $\mathrm{V}$ & Mild HP & $\mathrm{W}$ & 10 & 1 \\
\hline W & $\mathrm{Inv} \mathrm{Ca}$ & M & 95 & 3 \\
\hline $\mathrm{X}$ & DCIS-solid & M & 95 & 3 \\
\hline $2005 \mathrm{~A}$ & DCIS -micropap & M & 5 & 2 \\
\hline $\mathrm{C}$ & DCIS-micropap & M & 15 & 2 \\
\hline $\mathrm{D}$ & DCIS-micropap & M & 95 & $3^{* *}$ \\
\hline $387 \mathrm{~A}$ & DCIS-solid & M & 30 & 3 \\
\hline $\mathrm{E}$ & $\mathrm{N}$ & $\mathrm{W}$ & 0 & \\
\hline $775 \mathrm{G}$ & $\mathrm{N}$ & $\mathrm{W}$ & 40 & 1 \\
\hline $\mathrm{H}$ & Mod HP & $\mathrm{W}$ & 40 & 1 \\
\hline M & DCIS-solid & M & 0 & \\
\hline $2262 \mathrm{~A}$ & DCIS & M & 0 & \\
\hline B & DCIS & M & 0 & \\
\hline $\mathrm{D}$ & DCIS & M & 0 & \\
\hline $\mathrm{E}$ & DCIS & M & 0 & \\
\hline $\mathrm{F}$ & DCIS & M & 0 & \\
\hline $\mathrm{G}$ & $\mathrm{Inv} \mathrm{Ca}$ & M & 0 & \\
\hline
\end{tabular}

*Focal staining of moderate intensity in a portion of this normal lobule.

**Focal staining of a portion of micropapillary DCIS.

Inv Ca : invasive carcinoma; DCIS: ductal carcinoma in-situ; micropap : micropapillary; HP : hyperplasia; mod : moderate; met : metaplasia; $\mathrm{N}$ : normal breast epithelium; $\mathrm{M}:$ p53 mutation detected; W: wild type $p 53$.

tions. We were unable to detect truncation mutations with this antibody. This is in common with the findings of other investigators [13-15]. Thus, it appears that in general missense mutations stabilize the protein whereas truncated proteins, either alter the conformation of the protein so much that the antibody is unable to recognize the epitope, or the transcript and/or pro- tein product are unstable. The differences in staining characteristics between our cases could be explained by differences in the underlying mutations present. Rather than relating the intensity of staining to the type of mutation, others have found an association between the degree of immunohistochemical positivity for $\mathrm{p} 53$ and the histological grade of the lesion, with p53 staining being observed in high grade, comedo DCIS but not in low grade DCIS [16] and a greater frequency of positivity seen in high grade invasive carcinoma than in low grade invasive carcinoma [17, 18]. In our cases, intense staining could be found in DCIS in all cases with a missense mutation, regardless of grade.

We took advantage of information from a previous microdissection and SSCP/direct sequencing study [2] of the p53 mutational status in several of the epithelial lesions in 11 of the 12 cases. Knowledge of the mutational status of areas of DCIS and hyperplasia allows us to understand the staining patterns observed in the many epithelial areas in this analysis. In almost all instances, the results correlate well with the results obtained by immunohistochemistry. The earliest stage at which a confirmed mutation was detectable by both methods was DCIS. Two possible exceptions were found by immunohistochemistry. A portion of a normal lobule in case 490 that stained moderately strongly (suggesting a true mutation) was an unexpected finding. Whether this is the same mutation present in the invasive carcinoma or whether it is a second mutation present in the area perhaps due to a field effect that predisposes the local epithelium to missense mutations could not be determined as the sample was inadequate for genetic analysis. This lobule was at a distance from the invasive tumor making contiguous spread along the ductal system unlikely. In case 1857, a cluster of breast lobules adjacent to invasive carcinoma stained intensely but may merely represent contiguous spread of tumor cells. p53 mutations have been reported in benign breast tissue. In the study of Millikan et al. [19], three of 60 benign breast biopsies contained non-conservative $p 53$ mutations. These samples were a nonproliferative fibrocystic change, an area of papillomatous hyperplasia and a fibroadenoma. Thus, p53 mutations may sometimes occur at a histologically early stage and may indicate a greater propensity of the lesion to progress, although further studies are needed to address this.

Positive staining with anti p53 antibodies has been described in benign breast tissues [20, 21], however, the lesions showing positive staining have not often 
been subjected to mutational analysis. Younes et al., studied a group of 46 p53 positive breast biopsies that contained both benign and malignant lesions. They also observed strong intensity staining only in DCIS or invasive carcinoma. A recent study by Rohan et al. [22] concluded that p53 staining in benign breast biopsies is associated with an increased risk of future breast cancer. Thus, it may be that changes in p53 protein levels in mammary epithelium, whether due to increased levels of wild type or mutant protein, may be associated with the subsequent development of breast cancer. p53 has many functions in the cell including initiation of apoptosis and monitoring of the cell cycle. It is possible that increased levels of wild type $\mathrm{p} 53$ protein may represent a physiologic response to other molecular alterations, for example a disturbance in DNA replication fidelity or cell cycle control. Thus, weak intensity staining does not indicate an underlying p53 mutation but may signal an abnormality in the maintenance of genome stability and could still serve as a marker for high risk of neoplastic progression.

The presence of only focal staining in the case of extensive micropapillary DCIS with a missense mutation is more difficult to explain. All areas of histologically similar DCIS would be expected to stain strongly, but in this instance only a focal patch of staining was seen. Perhaps, despite the uniform presence of the same underlying p53 mutation, in three geographically distinct areas from this case, the p53 protein product is not stabilized or does not accumulate to detectable levels in some subpopulations of cells. These cells may contain additional genetic alterations.

Thus, it appears that while intense (score 3 out of 3 ) staining represents a missense mutation and weak (score 1 out of 3 ) staining correlates with wild type $\mathrm{p} 53$, the interface between these two groups lies within the intermediate (score 2 out of 3 ) zone. It is difficult to differentiate within this group because of the continuous nature of the staining intensity between and within cases, due in part to technical factors but probably also due to a gradual accumulation of $\mathrm{p} 53$ protein occurring in response to a mutation.

In the absence of a missense mutation it was possible to detect weak positive staining in some epithelial areas. We interpret this as an excess of wild type p53 protein for two reasons. Firstly, this type of staining was found to be similar in the normal lobules of cases with missense mutations and those with truncation mutations in their invasive tumors. Secondly, four normal lobules from these cases had previously been analyzed by SSCP/sequencing and no mutations were found. The significance of the presence of this weak staining in some early lesions is unclear.

In conclusion, we have demonstrated that strong intensity nuclear staining for p53 using the DO7 antip53 antibody is a sensitive and accurate marker of p53 missense mutations. In breast cancers known to harbor p53 missense mutations, immunohistochemical staining revealed that mutations could be uniformly identified in associated DCIS from the same resection specimen. No evidence was found to support a significant role for $p 53$ mutations in other associated non-invasive lesions such as epithelial hyperplasias. Lesser degrees of staining are often present in other non-invasive lesions, but do not seem to be associated with underlying $p 53$ mutations. Additional studies will be required to determine if these apparent physiologic increases in wild type p53 protein may serve as a high-risk marker for neoplastic progression.

\section{Acknowledgements}

This study was supported in part by grants from the Canadian Breast Cancer Research Initiative and the National Cancer Institute of Canada to M. Redston and I.L. Andrulis. S.J. Done was a Research Fellow of the National Cancer Institute of Canada supported by funds provided by the Terry Fox Run.

We thank the pathologists and surgeons of Mount Sinai Hospital for specimens and Kelvin So and Suzanna Tjan for excellent technical assistance.

\section{References}

1. Elledge RM, Allred DC: The p53 tumor suppressor gene in breast cancer. Breast Cancer Res Treat 32: 39-47, 1994

2. Done SJ, Arneson NC, Ozcelik H, Redston M, Andrulis IL: p53 mutations in mammary ductal carcinoma in situ but not in epithelial hyperplasias. Cancer Res 58: 785-789, 1998

3. Stephen CW, Helminen P, Lane DP: Characterisation of epitopes on human p53 using phage-displayed peptide libraries: insights into antibody-peptide interactions. J Mol Biol 248: 58-78, 1995

4. Umekita Y, Kobayashi K, Saheki T, Yoshida H: Nuclear accumulation of p53 protein correlates with mutations in the $p 53$ gene on archival paraffin-embedded tissues of human breast cancer. Jpn J Cancer Res 85: 825-830, 1994

5. Chakravarty G, Redkar A, Mittra I: A comparative study of detection of $p 53$ mutations in human breast cancer by flow cytometry, single-strand conformation polymorphism and genomic sequencing. Br J Cancer 74: 1181-1187, 1996

6. Lohmann D, Ruhri C, Schmitt M, Graeff H, Hofler H: Accumulation of $\mathrm{p} 53$ protein as an indicator for p53 gene 
mutation in breast cancer. Occurrence of false-positives and false-negatives. Diagn Mol Pathol 2: 36-41, 1993

7. Visscher DW, Sarkar FH, Shimoyama RK, Crissman JD: Correlation between p53 immunostaining patterns and gene sequence mutations in breast carcinoma. Diagn Mol Pathol 5: 187-193, 1996

8. MacGrogan G, Bonichon F, de Mascarel I, Trojani M, Durand M, Avril A, Coindre JM: Prognostic value of p53 in breast invasive ductal carcinoma: an immunohistochemical study on 942 cases. Breast Cancer Res Treat 36: 71-81, 1995

9. Silvestrini R, Benini E, Daidone MG, Veneroni S, Boracchi P, Cappelletti V, Di Fronzo G, Veronesi U: p53 as an independent prognostic marker in lymph node-negative breast cancer patients. J Natl Cancer Inst 85: 965-970, 1993

10. Pietilainen T, Lipponen P, Aaltomaa S, Eskelinen M, Kosma VM, Syrjanen K: Expression of p53 protein has no independent prognostic value in breast cancer. J Pathol 177: 225-232, 1995

11. Sjogren $\mathrm{S}$, Inganas $\mathrm{M}$, Norberg $\mathrm{T}$, Lindgren $\mathrm{A}$, Nordgren $\mathrm{H}$, Holmberg L, Bergh $\mathrm{J}$ : The p53 gene in breast cancer: prognostic value of complementary DNA sequencing versus immunohistochemistry. J Natl Cancer Inst 88: 173-182, 1996

12. Kovach JS, Hartmann A, Blaszyk H, Cunningham J, Schaid $\mathrm{D}$, Sommer SS: Mutation detection by highly sensitive methods indicates that p53 gene mutations in breast cancer can have important prognostic value. Proc Natl Acad Sci USA 93: 1093-1096, 1996

13. Kupryjanczyk J, Thor AD, Beauchamp R, Merritt V, Edgerton SM, Bell DA, Yandell DW: p53 gene mutations and protein accumulation in human ovarian cancer. Proc Natl Acad Sci USA 90: 4961-4965, 1993

14. Sommer SS, Cunningham J, McGovern RM, Saitoh S, Schroeder JJ, Wold LE, Kovach JS: Pattern of p53 gene mutations in breast cancers of women of the midwestern United States. J Natl Cancer Inst 84: 246-252, 1992

15. Hashimoto T, Tokuchi Y, Hayashi M, Kobayashi Y, Nishida K, Hayashi S, Ishikawa Y, Tsuchiya S, Nakagawa K, Hayashi J,
Tsuchiya E: p53 null mutations undetected by immunohistochemical staining predict a poor outcome with early-stage non-small cell lung carcinomas. Cancer Res 59: 5572-5577, 1999

16. O'Malley FP, Vnencak-Jones CL, Dupont WD, Parl F, Manning S, Page DL: p53 mutations are confined to the comedo type ductal carcinoma in situ of the breast. Immunohistochemical and sequencing data. Lab Invest 71: 67-72, 1994

17. Horne GM, Anderson JJ, Tiniakos DG, McIntosh GG, Thomas MD, Angus B, Henry JA, Lennard TW, Horne CH: p53 protein as a prognostic indicator in breast carcinoma: a comparison of four antibodies for immunohistochemistry. Br J Cancer 73: 29-35, 1996

18. Sasa M, Kondo K, Komaki K, Morimoto T, Monden Y: p53 alteration correlates with negative ER, negative $\mathrm{PgR}$, and high histologic grade in breast cancer. J Surg Oncol 56: 46-50, 1994

19. Millikan R, Hulka B, Thor A, Zhang Y, Edgerton S, Zhang X, Pei H, He M, Wold L, Melton LJ, Ballard D, Conway K, Liu ET: p53 mutations in benign breast tissue. J Clin Oncol 13: 2293-2300, 1995

20. Younes M, Lebovitz RM, Bommer KE, Cagle PT, Morton D, Khan S, Laucirica R: p53 accumulation in benign breast biopsy specimens. Hum Pathol 26: 155-158, 1995

21. Fischler DF, Sebek BA, Tubbs RR, Biscotti CV: p53 expression in intraductal epithelial hyperplasia, atypical intraductal hyperplasia, and intraductal carcinoma of the breast [letter; comment]. Histopathology 28: 93-94, 1996

22. Rohan TE, Hartwick W, Miller AB, Kandel RA: Immunohistochemical detection of c-erbB-2 and p53 in benign breast disease and breast cancer risk [see comments]. J Natl Cancer Inst 90: 1262-1269, 1998

Address for offprints and correspondence: Irene L. Andrulis, Samuel Lunenfeld Research Institute, Mount Sinai Hospital, 600 University Avenue, Room 984, Toronto, Ontario, Canada, M5G 1X5; Tel.: 416-586-8256; Fax: 416-586-8663; E-mail: andrulis@mshri.on.ca 